\title{
Les bambous gravés kanak dans les collections publiques françaises et à l'étranger
}

\section{Roger BOULAY}

\section{OpenEdition}

1 Journals

\section{Édition électronique}

URL : http://journals.openedition.org/jso/4912

DOI : $10.4000 /$ jso.4912

ISSN : $1760-7256$

\section{Éditeur}

Société des océanistes

\section{Édition imprimée}

Date de publication : 15 décembre 2008

Pagination : 318-319

ISBN : 978-2-85430-012-3

ISSN : 0300-953x

\section{Référence électronique}

Roger BOULAY, "Les bambous gravés kanak dans les collections publiques françaises et à l'étranger », Journal de la Société des Océanistes [En ligne], 126-127 | Année 2008, mis en ligne le 01 décembre 2008, consulté le 21 décembre 2020. URL : http://journals.openedition.org/jso/4912 ; DOI : https://doi.org/10.4000/jso.4912

\section{(c) (†) $\ominus$}

Journal de la société des océanistes est mis à disposition selon les termes de la Licence Creative Commons Attribution - Pas d'Utilisation Commerciale - Pas de Modification 4.0 International. 


\title{
Les bambous gravés kanak dans les collections publiques françaises et à l'étranger
}

par

\author{
Roger BOULAY*
}

Sur les 117 musées français (Boulay, 2007) possédant des collections océaniennes, une trentaine de musées conservent des bambous gravés kanak toujours liés à une collection plus diversifiée. On peut donc estimer leur total dans les collections publiques françaises à environ 150 bambous. Leurs dates de collecte (estimées en particulier d'après les dates connues du séjour sur place des collecteurs quand ils sont identifiés) les situent toutes après 1860, mais leur date d'arrivée dans les collections pouvant être beaucoup plus tardive. Cette date correspond précisément avec l'installation coloniale française qui s'officialise en 1853 (prise de possession de la Nouvelle-Calédonie). Les missionnaires qui, comme le révérend père Lambert (1900), commentèrent ces objets, sont présents en NouvelleCalédonie depuis 1843.

La très grande majorité des bambous ont des diamètres qui varient de 4 à 7 centimètres. Ce caractère botanique les rendrait contemporains de la pleine occupation européenne puisqu'il s'agirait d'une variété de bambou importée, tout spécialement dans la région de Canala (premier site choisi comme centre administratif sous Napoléon III). L'argument est consolidé par le fait qu'un bon nombre de sculptures faîtières représentées dans ces gravures peuvent être rapportées à cette même région dont la stylistique traditionnelle est très marquée et aisément identifiable (Boulay, 1993). Par ailleurs, on connaît la richesse en bambouseraies de la région de Thio,
Canala, Houaïlou (Guiart, 1963) : elle est le fait de l'acclimatation de cette variété importée. Toutefois, un bambou endémique (en xârâcùù $d \ddot{o}$ yee, « vrai bambou », Greslania sp. [voir MoyseFaurie et Néchéro-Jorédié, 1986]) d'un diamètre plus petit a été utilisé dans un certain nombre de cas. Ils portent, en général, un décor géométrique. On pourrait les considérer comme les aïeux des bambous gravés. L'irruption européenne a provoqué, sous l'influence de différents modèles importés, le maniement de la figuration (Boulay, 1997) dont le développement fut facilité par l'utilisation d'un support de diamètre plus important. Les noms de collecteurs, connus soit par leurs activités, soit par leurs publications, sont signalés.

\section{Listes des musées français ayant des bambous gravés}

Musée des Beaux-Arts d'Alençon : 1 - 1860, collecte Deplanche (Veillard et Deplanche, 1862).

Musée des Beaux-Arts d'Angoulême : 3 .

Musée Calvet, Avignon : 4 - collecte Mery.

Musée d'Aquitaine, Bordeaux : 1.

Musée savoisien, Chambery : 1 - collecte Vernaz (?) vers 1890.

Muséum Liais, Cherbourg : 2 - Grenier, 1889.

Musée-Château, Dieppe : 1.

Muséum d'histoire naturelle, Grenoble: 5 (Lavondès, 1990) dont 3 de Venge, 1893. 
Musée d'Art et d'Archéologie, Langres : 1 Gauvain (probabilité) avant 1858.

Muséum d'histoire naturelle, La Rochelle : 6.

Muséum d'histoire naturelle, Le Havre : 1 - collecte Le Mescam entre 1873 et 1902.

Muséum d'histoire naturelle, Lyon : 2.

Musée Dobré, Nantes : 1.

Musée d'histoire - Château des Ducs de Bretagne, Nantes : 2 - Gustave Glaumont avant 1891.

Nevers : 1 .

Muséum d'histoire naturelle, Nîmes : 4.

Musée de Nouvelle-Calédonie, Nouméa : 12.

Musée du Quai Branly, Paris : 70 - de Rochas (sans doute le plus ancien; avant 1860 [de Rochas, 1962]), Ardouin avant 1893.

Musée du Périgord, Périgueux : 7 ou 3 fiches collecte Lescure, avant 1889.

Musée des Beaux-Arts, Pithiviers : 1 ?

Musée des Beaux-Arts, Rennes : 8 - 1866-1905.

Hôtel Hebre de Saint-Clément, Rochefort : 2.

Muséum d'histoire naturelle, Rouen : 2 - Delaunay 1884 ; Knieder 1905.

Musée de la ville, Royan : 1.

Musée du Grand Donjon, Saint-Malo : 1.

Muséum d'histoire naturelle, Toulouse : 13 Savès. Balansa (très probable).

Musée du Cloître, Tulle : 1.

Les collections européennes, américaines, néozélandaises et australiennes

Adélaïde : 1.

Bâle : 4.

Berlin : 4.

Berne : 2 .

Cambridge : 1 .

Chicago : 1 .

Cologne : 2 .

Genève : 21 .

Hambourg : 2 .

Lausanne : 2 .

Leiden : 17

Londres : 9.

Neuchâtel : 1 .

New York (Metropolitan) : 1.
New York (American museum of Natural history) : ?.

Oxford : 2 .

Philadelphie : 9.

Rome Pigorini : 3 .

Rome Vatican : ?.

Saint Imier : 1 .

Stuttgart : 4 .

Sydney : ?.

Tervuren : 3 .

Zurich (Rieteberg) : ?.

Zurich (Université) : ?.

Wellington : 2 .

Wien : 3 .

Total du décompte $: 77^{1}$.

\section{BIBLIOGRAPHIE}

Boulay Roger, 1993. Le bambou gravé kanak, Marseilles, éd. Parenthèses et ADCK.

—, 1997. L'impact de l'imagerie religieuse sur les arts d'Océanie au XIX ${ }^{\mathrm{e}}$ siècle, in Océanie, curieux, navigateurs et savants, Paris, Éd. Somogy, pp. 113125.

—, 1996. Les bambous gravés figuratifs d'Océanie. Note sur le bouleversement de l'imagerie kanak au $\mathrm{XIX}^{\mathrm{e}}$ siècle, in Michèle Julien, Michel et Catherine Orliac (éds), Hommage à José Garanger. Mémoire de pierre, mémoire d'homme, Paris, Publications de la Sorbonne, pp. 301-311.

_, 2007. Annuaire des collections océaniennes dans les collections publiques françaises, en ligne sur la base Joconde, ministère de la Culture.

Guiart Jean, 1963. Océanie, Paris, Gallimard.

LAMBERT rév. père, 1900. Mours et superstitions des Néo-Calédoniens, Nouméa, réédition SEH 1980.

LAVONDÈs Anne, 1990. Vitrine des objets océaniens. Les collections du Muséum de Grenoble, Éd. Museum de Grenoble et ORSTOM.

Moyse-Faurie Claire et Marie-Adèle NÉCHÉRoJOREDIÉ, 1986. Dictionnaire xârâcùù-français, Nouméa, Édipop.

Vieillard E. et E. Deplanche, 1862. Essai sur la Nouvelle-Calédonie, Revue maritime et coloniale, T. VI., Paris.

Rochas Victor (de), 1862. La Nouvelle-Calédonie et ses habitants, Paris, éd. Sartorius. 